\title{
Agent societies and social networks for ubiquitous computing
}

\author{
Seungmin Rho $\cdot$ Naveen Chilamkurti $\cdot$ \\ Karim El Defrawy
}

Published online: 10 October 2013

(c) Springer-Verlag London 2013

\begin{abstract}
In ubiquitous computing environments, providing appropriate services and information to users at the right place in the right way is challenging for many reasons: different user interests, heterogeneous devices and services, dynamic networks, information overload, or differing privacy levels, for example. Agent technology is a paradigm expected to play an increasing role in complex computing environments, and due to the increasing popularity of social networking services, we expect to see the convergence of agent and social web technologies. The goal of this theme issue is to bring together state-of-the-art research contributions that examine the convergence of agent technologies and social networks for ubiquitous computing.
\end{abstract}

\section{Introduction}

We have received 24 manuscripts. Each manuscript was blindly reviewed by at least three reviewers consisting of guest editors and external reviewers. After the first and second review processes, eight manuscripts were finally selected to be included in this theme issue.

\footnotetext{
S. Rho $(\bowtie)$

Department of Multimedia, Sungkyul University,

Anyang-si, Korea

e-mail: smrho@sungkyul.ac.kr

N. Chilamkurti

Department of Computer Science and Engineering,

La Trobe University, Melbourne, Australia

e-mail: N.Chilamkurti@latrobe.edu.au

\section{K. E. Defrawy}

Information Systems and Sciences Lab, University

of California, Irvine, Irvine, CA, USA

e-mail: keldefra@uci.edu
}

In Sects. 2 and 3, we present a brief description about the agent technology and intelligent social network technology issues in ubiquitous computing environment, respectively. Section 4 describes other issues for personal and ubiquitous computing. In the last section, we conclude the paper with some observations and future work.

\section{Agent technologies for ubiquitous computing}

The first paper entitled "ALCA: Agent Learning Based Clustering in Vehicular Ad Hoc Networks" by Kumar et al. [1] proposes new agent-based clustering and routing in VANETs and also present agent-based learning algorithm for clustering. Agents learn from the environment in which they are deployed, and accordingly their action performed is rewarded or penalized with certain values. Each agent performs its task in collaboration with the other agents, i.e., agents communicate with each other in collaborative manner for information sharing.

The second paper entitled "Semi-automatic Construction of Domain Ontology for Agent Reasoning" by Rho et al. [2] presents a hybrid method to automatically extract relations from domain documents which combines a named relation approach and an unnamed relation approach. They used soft matching method to recognize various context forms of the relation in a sentence to generalize patterns and unnamed relation approach to assign useful relation names to unnamed-group relations on $58.33 \%$ precision.

\section{Social networks and intelligent technologies for ubiquitous computing}

Third paper entitled "Recommendation of Optimized Information Seeking Process Based on the Similarity of 
User Access Behavior Patterns" by Chen et al. [3] proposes an integrated adaptive framework to support and facilitate individualized recommendation based on the Gradual Adaptation Model that gradually adapts to a target user's transition of needs and behaviors of information access, including various search related activities, over different time spans.

Next paper entitled "Social Relation based Dynamic Team Organization by Context-aware Matchmaking" by Chang et al. [4] proposes a way of maintaining team in spite of the member's modification in the dynamically changing situation. In order to achieve this goal, they employed an abstract layer, which provides roles to the team by hiding the members, between team and members.

\section{Other issues in ubiquitous computing}

Fifth paper entitled "Genetic Algorithm for Effective Open Port Selection for a Web Filter" by Yeo et al. [5] proposes a method for networking-based application that addresses the problem of selecting ports to leave open for any given web filter. For this, they applied a genetic algorithm to choose the appropriate ports as part of the final result which is encoded into chromosomes using value encoding.

Next paper entitled "RFID based Indoor Location Tracking to Ensure the Safety of the Elderly in Smart Home Environments" by Park et al. [6] proposes an indoor U-Healthcare system that uses radio-frequency identification (RFID) technology to accurately locate and track the elderly. The proposed system provides real-time monitoring of elderly people's whereabouts. In addition, it analyzes their locations in association with time slots and the length of time they stay in the same place, thus inferring information such as movement patterns, ranges, and frequencies.

Another paper entitled "Evaluation of Messaging Middleware for High Performance Cloud Computing" by Doallo et al. [7] presents an evaluation of high-performance computing message-passing middleware on a cloud computing infrastructure, Amazon EC2 cluster compute instances, equipped with 10 gigabit Ethernet. The analysis of the experimental results, confronted with a similar testbed, has shown the significant impact that virtualized environments still have on communications performance, which demands more efficient communication middleware support to get over current cloud network limitations.

Eighth paper entitled "Modeling User-Generated Contents: An Intelligent State Machine for User-Centric Search Support" by Shih et al. [8] proposes an intelligent state machine as a hybridization of graph model (Document
Graph) and petri-net model (Document Sensitive Petri-Net) to make efficient use of user-generated contents. It is utilized to clarify the vague usage scenario between usergenerated contents, such as discussions, posts, and to identify correlations and experiences within them.

Next paper entitled "Semantic Web service discovery: state-of-the-art and research challenges" by Ngan et al. [9] provides an extensive review of Semantic Web Service (SWS) discovery, highlighting the state-of-the-art approaches, the key semantic formalisms employed, as well as benchmarks and testbeds for performance evaluation. Defining a generic framework for semantic service discovery, they describe the key tasks and criteria involved in agent-based computing. They also gave the detailed comparison of the popular discovery systems with a discussion on tradeoffs between existing approaches.

The last paper entitled "Efficient Journaling Writeback Schemes for Reliable and High Performance Storage Systems" by Lim et al. [10] presents an efficient writeback schemes called defragmented writeback (DFW) that enables guaranteeing throughput in high-performance storage systems. It reduces positioning time of storage devices in writing workloads and thus enables fast writeback in storage systems. They consider both of storage media in designing DFW scheme, traditional rotating disk and emerging solid state disks.

\section{Conclusion}

Finally, our special thanks go to Prof. Peter Thomas and all editorial staffs for their valuable supports throughout the preparation and publication of this theme issue. We would like to thank all authors for their contributions to this theme issue. We also extend our thanks to the following external reviewers for their excellent help in reviewing the manuscripts.

\section{References}

1. Kumar N, Chilamkurti N, Park JH (2012) LCA: agent learning based clustering in vehicular ad hoc networks. Pers Ubiquitous Comput. doi:10.1007/s00779-012-0600-8

2. Choi I, Rho S, Kim M (2012) Semi-automatic construction of domain ontology for agent reasoning. Pers Ubiquitous Comput. doi:10.1007/s00779-012-0606-2

3. Chen J, Zhou X, Jin Q (2012) Recommendation of optimized information seeking process based on the similarity of user access behavior patterns. Pers Ubiquitous Comput. doi:10.1007/s00779012-0601-7

4. Lee K, Kim J, Rho S, Chang H (2012) Social relation-based dynamic team organization by context-aware matchmaking. Pers Ubiquitous Comput. doi:10.1007/s00779-012-0608-0 
5. Hussain S, Olayemi A, Yeo S-S (2012) Genetic algorithm for effective open port selection for a web filter. Pers Ubiquitous Comput. doi:10.1007/s00779-012-0602-6

6. Kim S-C, Jeong Y-S, Park S-O (2012) RFID-based indoor location tracking to ensure the safety of the elderly in smart home environments. Pers Ubiquitous Comput. doi:10.1007/s00779012-0604-4

7. Expósito RR, Taboada GL, Ramos S, Touriño J, Doallo R (2012) Evaluation of Messaging Middleware for High Performance Cloud Computing. Pers Ubiquitous Comput. doi:10.1007/s00779012-0605-3
8. Yen NY, James J, Jin Q, Shih TK (2012) Modeling User-Generated Contents: An Intelligent State Machine for User-Centric Search Support. Pers Ubiquitous Comput. doi:10.1007/s00779012-0607-1

9. Ngan LD, Kanagasabai R (2012) Semantic Web service discovery: state-of-the-art and research challenges. Pers Ubiquitous Comput. doi:10.1007/s00779-012-0609-z

10. Lim S-H, Choi HJ, Park D-S (2012) Efficient Journaling Writeback Schemes for Reliable and High Performance Storage Systems. Pers Ubiquitous Comput. doi:10.1007/s00779-012-0603-5 